\title{
Gender and Age Differences in Toe Muscle Strength in Childhood and the Relationship between Toe Muscle Strength and Basic Motor Skills
}

\author{
Sakiko UKITA ${ }^{1,2, *}$, Kaoru TSUYAMA ${ }^{3}$ \\ ${ }^{1}$ Doctoral Degree Program, Nippon Sport Science University, Tokyo, Japan \\ ${ }^{2}$ College of Education, Kanto Gakuin University, Kanagawa Japan \\ ${ }^{3}$ Faculty of Sport Science, Nippon Sport Science University, Kanagawa Japan \\ *Corresponding author: saki0911@kanto-gakuin.ac.jp
}

\begin{abstract}
In this study, we examined gender differences and age-related changes in toe muscle strength in childhood as well as the relationship between toe muscle strength and basic motor skills. The subjects included 288 children aged between 6 and 10 years who were enrolled in elementary school. Measurements were conducted for toe muscle strength, long-distance throw, $50 \mathrm{~m}$ running time, and standing long jump distance. Toe muscle strength develops with age throughout childhood; no differences were observed in toe muscle strength between girls and boys; however, a significant correlation was observed between toe muscle strength and each of long-distance throw, $50 \mathrm{~m}$ running time, and standing long jump distance. There was also a significant partial correlation (eliminating the influence of age) between toe muscle strength and long-throw distance, $50 \mathrm{~m}$ running time, and standing long-jump distance for both boys and girls. The results indicate that toe muscle strength tends to develop with age, but there are no gender differences. Results also suggested that toe muscle strength is significantly related to basic motor skills.
\end{abstract}

Keywords: toe muscle strength, childhood, motor skills

Cite This Article: Sakiko UKITA, and Kaoru TSUYAMA, "Gender and Age Differences in Toe Muscle Strength in Childhood and the Relationship between Toe Muscle Strength and Basic Motor Skills" American Journal of Sports Science and Medicine, vol. 5, no. 3 (2017): 48-52. doi: 10.12691/ajssm-5-3-2.

\section{Introduction}

The importance of the function of the toes with regard to humans walking has been noted from the perspective of an increase in weight support area and propulsion force $[1,2]$. Other important functions of toes include posture retention, initial kicking when starting to walk, agile response to movements in the left and right directions, support on slopes and rough terrain, and standing on tiptoes [3]. To date, most reports on the functions of toes have focused on young, middle-aged, or elderly people. For example, Kito et al [4] found that a group of elderly people who had had experiences of falling had less toe muscle strength when compared with a group of elderly people with no experience of falling. However, although the toes' functions are important not only for adults and the elderly but also for children during early childhood and elementary school age, only a few studies of this nature have been conducted on children, and many aspects have not been clarified. Since lower limb muscle thickness tends to develop with age during the first half of elementary school, it is possible that toe muscle strength develops with age. Conversely, as no gender differences have been recognized in lower limb muscle thickness, it is possible that there are no gender differences in toe muscle strength during childhood.
Meanwhile, the agonist muscle of toe muscle strength is regarded as the CSA of the inner portion of the intrinsic muscles at the bottom of the foot, namely, the flexor pollicis brevis muscle, the flexor digitorum brevis muscle, the quadratus plantaris, the lumbrical muscles, and the abductor hallucis muscle [5]. The development of toe muscle strength contributes to the improvement of physical ability [6], and it has been shown that a low level of toe muscle strength is associated with a high risk of falling [7]. Although basic movements, including running, hopping, and throwing, develop remarkably well during the period from infancy to childhood, the kicking up movement is involved in these motion movements. Therefore, there may be a significant relationship between toe muscle strength and running and hopping performance.

Thus, the purpose of this study was to examine gender and age differences in toe muscle strength in childhood as well as the relationship between toe muscle strength and basic motor skills.

\section{Methods}

\subsection{Subjects}

A total of 288 subjects participated in the study; they included 20 and 21 six-year-old boys and girls, 
respectively; 37 and 35 seven-year-old boys and girls, respectively; 23 and 15 eight-year-old boys and girls, respectively; 23 and 24 nine-year-old boys and girls, respectively; and 48 and 42 ten-year-old boys and girls, respectively.

In addition, the study was conducted with the approval of the Kanto Gakuin University and Nippon Sport Science University's Human Research Ethics Review Committee (Approval Number: Person 2014-1-1, Number 015-H42). Furthermore, consent to participate in the study was obtained after the purpose of this study was explained verbally to the subjects and in writing to their parents before the study commenced.

\subsection{Measurement Items}

\section{(1) Method of measuring motor skills}

1. Evaluation of the ability to throw (Measurement of the long-distance throw)

A circle $2 \mathrm{~m}$ in diameter was drawn on the ground, and as shown in Figure 1, a straight line was drawn from the center of the circle so as to form a central angle of 30 degrees; within this, a concentric circular arc at a $1 \mathrm{~m}$ interval was drawn. The subjects were required to throw a softball (with an outer circumference of $26.2 \mathrm{~cm}-27.2 \mathrm{~cm}$, weight $136 \mathrm{~g}-146 \mathrm{~g}$ ) from within the circle. The subjects were cautioned not to step on or go over the circle and instructed to ensure that the ball did not go out of the 30-degree range. If the subjects did not abide by the aforementioned precautions, the measurements were conducted again. With respect to the recording of measurements, the distance to the point where the ball dropped was measured in accordance with the arc drawn at a $1-\mathrm{m}$ interval. The recorded representative value was the better of the values of the two tries conducted, with the meter as the unit.

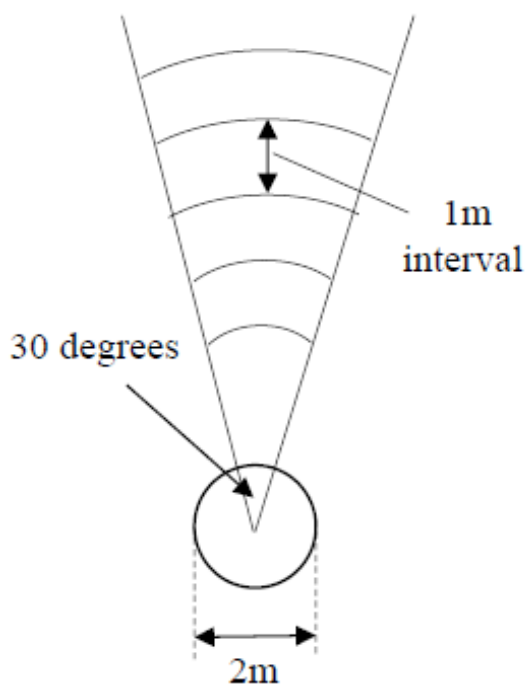

Figure 1. Throw Range of the Long Throw Distance Measurement

2. Evaluation of running ability (measurement of $50 \mathrm{~m}$ running time)

A straight track of $50 \mathrm{~m}$ with a course width of $125 \mathrm{~cm}$ was set up on the ground (refer to Figure 2). The width of the line was $5 \mathrm{~cm}$. The subjects responded to the cue "Ready-Go" to commence with a standing start. The time taken for each subject to reach the goal line was measured.
The recording unit was $1 / 10$ of a second and a time of less than $1 / 10$ seconds was rounded off. Measurements were conducted once.

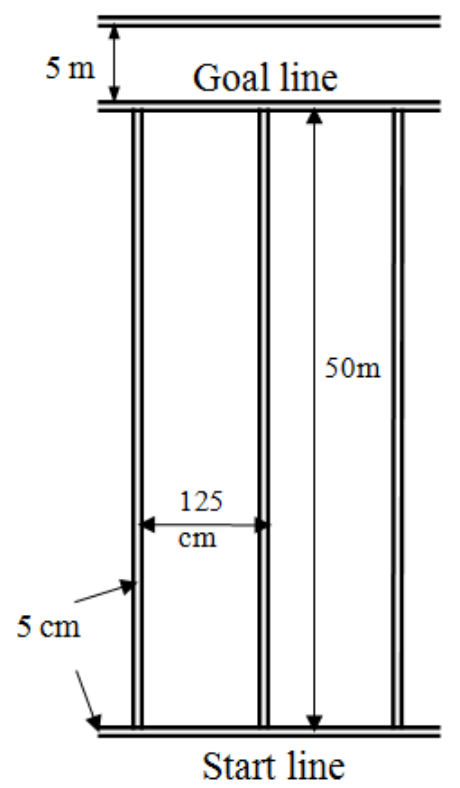

Figure 2. Straight Track of $50 \mathrm{~m}$ Running Time Measurement

3. Evaluation of jumping ability (Measurement of standing long jump distance)

Mats developed for standing long jump were prepared (TOEILIGHT CO., LTD., T-2598). The subjects prepared themselves by standing on the mat with their legs slightly apart and their toes aligned with the front edge of the take-off line and jumped when they were ready. At the time of the jump, they were instructed to jump forward as far as possible. The distance from the take-off line to the heel of the foot closest to the take-off line was measured and recorded twice; the greater value was used as a representative value.

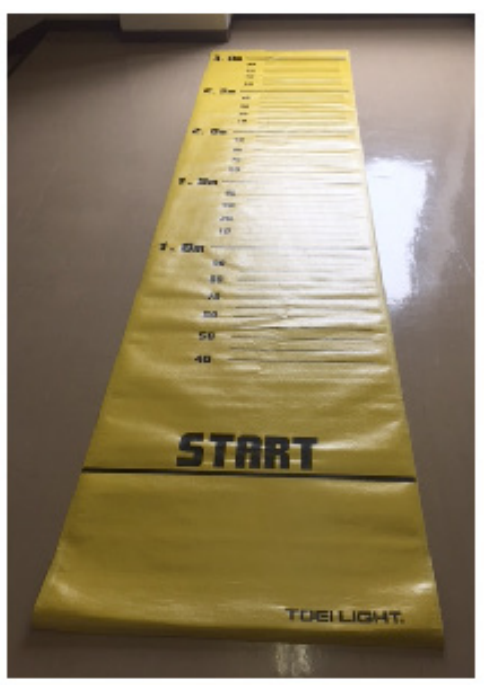

Figure 3. Mats for Standing Long Jump

\section{(2) Measurement of toe muscle strength}

Toe muscle strength was measured using the toe flexor dynamometer (T.K.K.3361 manufactured by Takei Scientific Instruments Co., Ltd.) and according to measurement methods used in previous research [8]. Measurements 
were conducted with participants seated with their hip joints at 90 degrees, knee joints at 90 degrees in a bending position, and ankle joint dorsiflexion at 0 degrees. Subjects were instructed to put both their hands on their knees during measurement, keep the upper body from leaning on the chair, put their five toes on the grip bar of the toe muscle strength meter, and flex their toes with maximum effort. The toe muscle strength of the left and right legs was measured twice each. The average of the greater of the values of the left and right legs was adopted as the representative value (Refer to Figure 4).

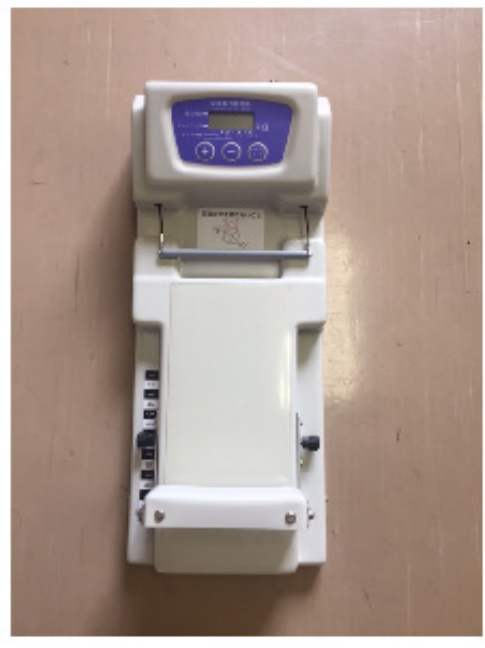

Firuge 4. Toe Flexor Dynamometer

\subsection{Statistical Processing}

To test the difference between age-wise and genderwise average values, a two-factor (gender $\times$ age) variance analysis where both factors do not have correspondence was adopted. When a significant interaction or primary effect was observed, the Tukey method was used and a multiple comparison test was performed. The relationship between the variables was examined using the Pearson correlation coefficient and the partial correlation coefficient. The significance level was set at a risk rate of less than $5 \%$.

\section{Results}

Table 1 shows the results of age-wise and gender-wise averages and that of the two-factor variance analysis of toe muscle strength. Significant primary effects were observed only for age, and the results of multiple comparison testing showed that toe muscle strength was greater in boys; 8-, 9-, and 10-year-olds were stronger than 6-year-olds, and 9- and 10-year-olds were stronger than 7- and 8-year-olds. Among girls, 8-, 9-, and 10-yearolds were stronger than 6-year-olds, 9- and 10-year-olds were stronger than 7-year-olds, and 10-year-olds were stronger than 8-year-olds.

Table 2 shows the results of the age-wise and gender-wise two-factor variance analysis of long-throw distance, $50 \mathrm{~m}$ running time, and standing long jump distance. Longthrow distance was observed to significantly interact with both factors, and a significant primary effect was observed for the age factor for all items. A significant primary effect was also observed for the gender factor in longthrow distance, and the results of multiple comparison testing showed a significant difference in the average values between boys and girls aged 7, 8, 9, and 10 years, with boys having a greater long-throw distance. No significant interaction was observed for $50 \mathrm{~m}$ running time, and a significant primary effect was observed for the age factor for all items. No significant interaction was observed for standing long-jump distance, but a significant primary effect was observed for the age and gender factors for all items. However, the results of multiple comparison testing revealed no significant differences in the average values between boys and girls, irrespective of age. The results of multiple comparison testing are presented in a note in Table 2.

Table 3 shows the partial correlation coefficient and the correlation coefficient of variable interactions between toe muscle strength and long-throw distance, $50 \mathrm{~m}$ running time, and standing long jump distance. The correlation coefficient was found to be moderately significantly correlated with all exercise-capacity tests. The partial correlation coefficient was also lower than the correlation coefficient, but both were significant.

Table 1. Age-wise and Gender-wise Average Values of Toe Muscle Strength and Results of the Two-Factor Variance Analysis

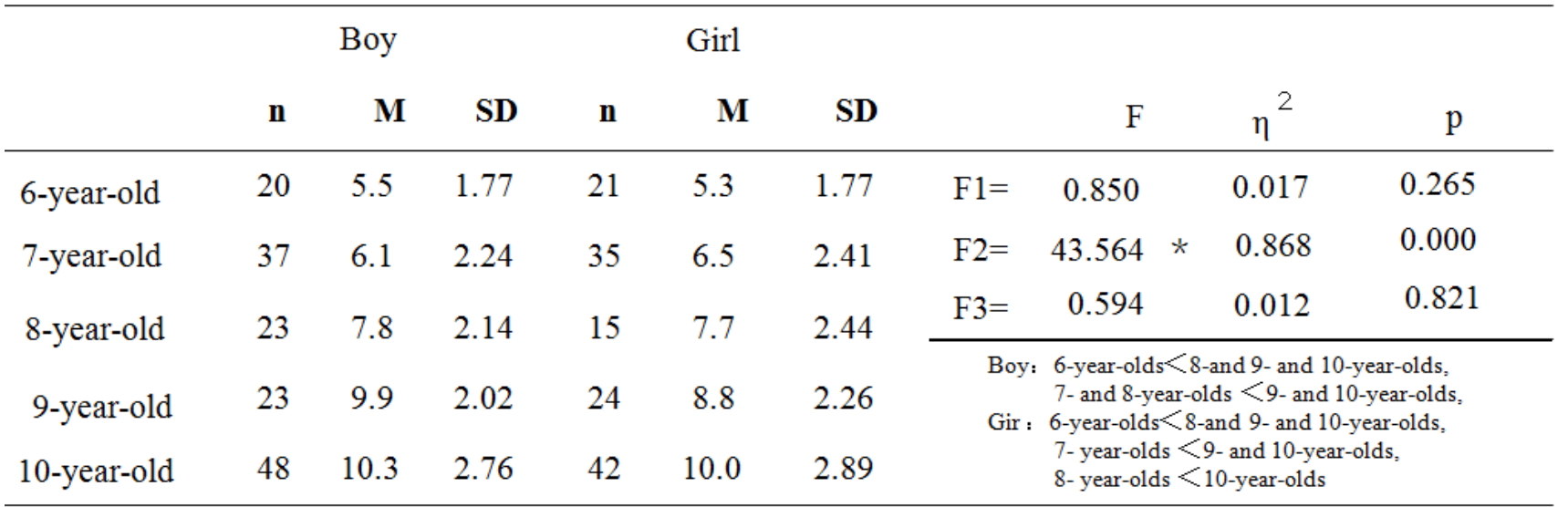

Note) $\star: \mathrm{p}<0.05, \mathrm{~F} 1$ : Gender factor, F2: Age factor, F3: Interaction, 
Table 2. Results of the Age-Wise and Gender-Wise Two-Factor Variance Analysis of Long-Throw Distance, 50m Running time and Standing Long jump Distance

\begin{tabular}{|c|c|c|c|c|c|c|c|c|c|c|c|c|c|c|c|}
\hline & \multicolumn{4}{|c|}{ Long-Throw Distance (m) } & \multicolumn{4}{|c|}{ 50m Running time ( $\mathrm{sec}$ ) } & \multicolumn{4}{|c|}{ Standing Long jump Distance ( Cm ) } & & \multirow{3}{*}{$\mathrm{F}$} & \multirow{3}{*}{$\eta^{2}$} \\
\hline & \multicolumn{2}{|c|}{ Boy } & \multicolumn{2}{|c|}{ Girl } & \multicolumn{2}{|c|}{ Boy } & \multicolumn{2}{|c|}{ Girl } & \multicolumn{2}{|c|}{ Boy } & \multicolumn{2}{|c|}{ Girl } & & & \\
\hline & $\mathbf{M}$ & SD & $\mathbf{M}$ & SD & $\mathbf{M}$ & SD & M & SD & $\mathbf{M}$ & SD & $\mathbf{M}$ & SD & & & \\
\hline 6-year-old & 7.6 & 1.03 & 5.7 & 1.00 & 12.1 & 0.24 & 13.0 & 0.23 & 123.0 & 17.95 & 112.6 & 20.47 & $F=$ & 50.492 & 3.654 \\
\hline 7-year-old & 9.7 & 0.76 & 7.1 & 0.78 & 11.2 & 0.17 & 11.2 & 0.18 & 120.8 & 21.87 & 120.3 & 15.55 & 营 $\mathrm{E}=$ & 41.453 & 3.000 \\
\hline 8-year-old & 14.3 & 0.96 & 9.3 & 1.19 & 10.3 & 0.22 & 10.7 & 0.27 & 138.7 & 15.46 & 134.0 & 11.53 & 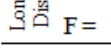 & 3.657 & 0.265 \\
\hline 9-year-old & 14.5 & 0.96 & 10.7 & 0.94 & 10.3 & 0.22 & 10.0 & 0.21 & 144.8 & 21.45 & 140.6 & 17.96 & 刍 $\mathrm{F}=$ & 3.322 & 0.013 \\
\hline \multirow[t]{5}{*}{ 10-year-old } & 19.6 & 0.66 & 12.4 & 0.71 & 9.5 & 0.15 & 9.8 & 0.16 & 150.2 & 17.33 & 146.9 & 20.43 & $F=$ & 62.537 & 0.237 \\
\hline & & & & & & & & & & & & & 点首 $\mathrm{F}=$ & 2.361 & 0.009 \\
\hline & & & & & & & & & & & & & 总 总 $\mathrm{F}=$ & 3.932 & 4.627 \\
\hline & & & & & & & & & & & & & F= & 33.628 & * $\quad 39.570$ \\
\hline & & & & & & & & & & & & & 基具 $\mathrm{F}=$ & 0.471 & 0.554 \\
\hline
\end{tabular}

Note) $火: p<0.05$, F1: Gender factor, F2: Age factor, F3: Interaction, See Table 1 for the number of people,

Post-hoc analysis of gender differences : Long-Throw Distance : 7-and 8-and 9-and 10 year-old Girls $<$ Boys Standing Long jump Distance : n.s

Post-hoc analysis of age differences : Long-Throw Distance : Boys : 6 - and 7-year-olds $<8$ - and 9 -and 10 -year-olds, 8 -and 9 -year-olds $<10$-year-olds Girls : 6 - and 7-year-olds $<9$-and 10 -year-olds,

50m Running time : Boys : 6-year-olds $>7$-and 8 -and 9-and 10-year-olds, 7-year-olds $>8$-and 9-year-olds $>10$-year-olds Girls : 6 -year-olds $>7$ - and 8 - and 9 - and 10 -year-olds,

7 -year-olds $>9$-and 10-year-olds, 8 -year-olds $>10$-year-olds

Standing Long jump Distance : Boys : 6-and 7-year-olds $<8$ - and 9 -and 10 -year-olds

Girls : 6-year-olds $<8$-and 9 -and 10 -year-olds, 7 -year-olds $<9$-and 10 -year-olds

Table 3. Correlation Coefficients and Partial Correlation Coefficients Between Toe Muscle Strength and Each of Long-Throw Distance and $50 \mathrm{~m}$ Running Time and Standing Long jump Distance

\begin{tabular}{lccccc}
\hline & \multicolumn{3}{c}{ Correlation Coefficient } & \multicolumn{2}{c}{ Partial Correlation } \\
& Boy & Girl & Boy & Girl \\
\hline Long-Throw Distance & $0.597 *$ & $0.531 *$ & $0.331 *$ & $0.285 *$ \\
50m Running time & -0.547 & $*$ & $-0.576 *$ & $-0.243 *$ & $-0.298 *$ \\
Standing Long jump Distance & $0.595 *$ & $0.586 *$ & $0.388 *$ & $0.351 *$ \\
\hline
\end{tabular}

Note) $*: \mathrm{p}<0.05$.

\section{Discussion}

Although there were no gender differences seen for toe muscle strength, the difference tended to increase with advancement in age. With advancement in age, muscle mass increased, and muscle strength also developed along with it. However, conversely, in adolescence, this tendency was more prominent in men than it was in women [9]. As the subjects of this study were pre-adolescent children, there were no gender differences in toe muscle strength. It can be inferred from this that there were also no gender differences in the developmental tendencies arising from advancement in age. Conversely, for long-throw distance, $50 \mathrm{~m}$ running time, and standing long jump distance, there were changes with advancement in age that were similar to those for toe muscle strength even though gender differences were observed only for long-throw distance. The $50 \mathrm{~m}$ running time and standing long jump distance, for which the prime movers are the muscle groups of the lower limbs, were considered to potentially have the same developmental tendencies and gender difference tendencies with advancement in age with regard to toe muscle strength (i.e., the muscle strength of the lower limbs).

An examination of the relationship between measurement items for toe muscle strength revealed that there was a significant relationship. In previous research, it has been reported that the toe muscle strength of children influences the field record of physical fitness tests [2]. It also shows that for toe muscle strength and standing long jump distance, it is the toe that is the site of action on the ground and that the characteristics of this muscle strength performance influences jumping force [10]. It may also influence the items related to toe muscle strength and lower limbs (standing long jump distance, repetitive horizontal jump, and $50 \mathrm{~m}$ running time) [2]. Further, it has been reported that toe muscle strength is important for forward propulsion force when kicking out during the running motion and in the stability of the shift in the center of gravity as well [11]. Noda [12], notes that important functions of the toe include holding the posture, initial kicking when starting to walk, agile response to movements in the left and right directions, response on slopes and rough terrain, and tensing while standing on tiptoes.

It is believed that as toe muscle strength exerts considerable influence on the securing of forward propulsion force when running, on the step-and-kick action when throwing, and on the taking-off action when leaping, a relationship between toe muscle strength and long-throw distance, $50 \mathrm{~m}$ running time, and standing long jump distance exists.

\section{Summary}

In this study, we examined the relationship between the development of toe muscle strength in children with advancement in age and physical fitness and motor skills. The results are as follows: Toe muscle strength tends to develop with advancement in age during childhood; however, no gender differences were observed. Further, toe muscle strength was related to long-throw distance, 50 $\mathrm{m}$ running time, and standing long jump distance. Even in 
the partial correlation coefficient eliminating the influence of age, in both boys and girls, toe muscle strength was related to long-throw distance, $50 \mathrm{~m}$ running time, and standing long-jump distance. It was suggested that toe muscle strength is closely related to basic motor skills.

\section{Acknowledgments}

I would like to express my sincere gratitude to the principal, teachers, children, and parents of Kanto Gakuin Mutsuura Elementary School for their support and cooperation. Also, this study was conducted with the assistance of teacher research grants from the Society of Human and Environmental Studies, College of Human and Environmental Studies, Kanto Gakuin University for the academic years 2015 and 2016.

\section{References}

[1] Uritani D, Fukumoto T, Matsumoto D, and Shima M, "Uritani et al. Reference values for toe grip strength among Japanese adults aged 20 to 79 years," Journal of Foot and Ankle Research, 7: 1-6. 2014.

[2] Morita N, Yamauchi J, Kurihara T, Fukuoka R, Otsuka M, Okuda T, Ishizawa N, Nakajima T, Nakamichi R, Matsuno S, Kamiie S, Shide N, Kambayshi I, and Shinkaiya H, "Toe Flexor Strength and Foot Arch Height in Children," J. Phys. Ther. Sci.27. 3533-3536. 2015.
[3] Soma M, Igarashi T, Kudo W, Nakae H, and Abiko T, "Regarding the Influence of Toe Grip Force Training on Functional Reach Test Or On Width of Maximum Stride and Walking Ability," Japanese Journal of Health Promotion and Physical Therapy, 2(2). 59-63. 2012.

[4] Kito N, Hidetoshi I, Megumi M "The Effect of Toe Training as Prevention of Falling in Elderly," Physical Therapy Japan, 28(7). 313-319, 2001.

[5] Kurihara T, Yamauchi J, Otsuka M, Tottori N, Hashimoto T, and Isaka $\mathrm{T}$, "Maximum toe flexor muscle strength and quantitative analysis of human plantar intrinsic and extrinsic muscles by a magnetic resonance imaging technique," J Foot Ankle Res.7.26. 2014.

[6] Goldmann JP, Sanno M, Willwacher S, Heinrich K, and Brüggemann Gp. "The Potential of toe flexor muscles to enhance performance," J Sports Sci. 31(4). 424-433. 2012.

[7] Mickle KJ, Munro BJ, Load SR, Menz HB, and Steele JR, "Toe weakness and deformity increase the risk of falls in older people, " Clin Biomech (Bristol, Avon);24. 787-791. 2009.

[8] Soma M, Murata S, Kai Y, Nakae H, and Satou Y, "An Examination of Limb Position for Measuring Toe-grip Strength," J Phys Ther Sci. 26(12). 1955-1957. 2014.

[9] Katić R1, Pavić R, Cavala M, “Quantitative sex differentations of motor abilities in children aged 11-14," Coll Antropol. 37(1). 81-86. 2013

[10] Fukuoka R, Morita N, Yamauchi J, and Kurihara T, "The Relationship Between Toe Muscle Strength and Grip Force and Standing Long Jump in Children," The Japanese Journal of Physical Fitness and Sports Medicine, 62(6). 552. 2013.

[11] Yamada K, Sudo A, "The Relationship Between Toe Grip Force and Sprinting Speed," The Society of Physical Therapy Science, 30(4). 519-521.2015.

[12] Noda Y, "Body as Seen from the Sole of the Foot, "Kodansha, Tokyo, 95-96. 1998. 